\title{
Four-wave neutron-resonance spin echo
}

\author{
S. V. Grigoriev, ${ }^{1,2}$ W. H. Kraan, ${ }^{1}$ and M. Th. Rekveldt ${ }^{1}$ \\ ${ }^{1}$ Interfacultair Reactor Instituut, TU-Delft, $2629 \mathrm{JB}$ Delft, The Netherlands \\ ${ }^{2}$ Petersburg Nuclear Physics Institute, Gatchina, St. Petersburg 188300, Russia
}

(Received 21 December 2003; published 21 April 2004)

\begin{abstract}
We develop a technique of scattering from many-body systems. It is based on the principle of the neutron spin echo (SE), where a neutron wave in the magnetic field splits into two waves, which are separated in space or in time after propagation in this field. The neutron thus prepared as a probe passes through the sample to test its properties on a space $R$ or time $t$ scale. This separation in space or in time can be measured using coherence of these two waves as a phase shift $\phi$ between them. These two waves are collected or focused and compensated by the SE technique in order to compare their phases after interaction with the sample. In this way one studies interference between these waves and thus can directly measure the pair-correlation function in space or in time. Instead of two-wave SE we propose to realize the four-wave neutron-resonance spin-echo (NRSE). In our experiments, spin precession produced by a couple of the neutron-resonance coils in one arm is compensated by an identical couple of other NR coils in a second arm of a spin-echo machine. The neutron spin-flip probability $\rho$ in the resonance coils is a key parameter of the NRSE arm. The limiting cases, $\rho=0$ and $\rho=1$, provide, in quantum terms, a two-level-two-wave $k$ splitting of the neutron and result in the separation of the split waves into two different lengths in space $\left(R_{1}, R_{2}\right)$ or in time $\left(t_{1}, t_{2}\right)$. These two cases correspond to Larmor precession with phase $\phi_{1}$ in the static magnetic fields of the NR flippers or to NRSE precession with $\phi_{2}$, respectively. The intermediate case, $0<\rho<1$, provides a four-level-four-wave $k$ splitting of the neutron with the corresponding separations in space $\left(R_{1}, R_{2}, R_{3}\right)$ or in time $\left(t_{1}, t_{2}, t_{3}\right)$. The interference of each pair of waves after compensation results in three different echos with phases $\phi_{1}, \phi_{2}$, and $\phi_{3}=\left(\phi_{1}+\phi_{2}\right) / 2$. Focusing or compensating all four waves into a single point of the phase-of-waves diagram produces quantum interference of all newly created waves. This task of focusing is experimentally performed. Different options for the compensation are discussed. The experiment opens the possibility to measure a composite correlation function, combined from several pair-correlation functions.
\end{abstract}

DOI: 10.1103/PhysRevA.69.043615

PACS number(s): 03.75.Dg, 42.87.Bg

\section{INTRODUCTION}

Neutron-resonance spin echo (NRSE) is a new, quickly progressing method for manipulation of the spin in neutron beam scattering experiments [1-3]. The magnetic-field precession area of the conventional spin echo (SE) technique [4] is replaced in this method by a pair of resonance coils, placed in field $B_{0}$ and operated at frequency $\omega_{\mathrm{RF}}$, satisfying the resonance condition $\omega_{\mathrm{RF}}=\gamma B_{0}$ and separated by a distance $L$ of zero magnetic field. In terms of precession, this pair of RF coils simulates a DC field integral $2 \gamma_{n} B_{0} L$, where $\gamma_{n}$ is the neutron gyromagnetic ratio. This is understood if Larmor precession is considered as interference between two superposed neutron waves. These waves come into existence because in the static magnetic field the incoming neutron wave with momentum $\vec{k}$ is split into two waves with different momenta $\vec{k}_{+}$and $\vec{k}_{-}$. The actions of the resonance coil on the neutron wave may be counted as (1) splitting in the permanent field $B_{0}$; (2) spin flipping in the resonance coil; (3) a doubling of the splitting when neutron leaves the static magnetic field $B_{0}$, due to the energy conservation law. This precession, or $k$ splitting, lasts until the second resonance coil, located at a distance $L$ from the first one, which cancels the splitting and therefore stops the precession. These coils together and the distance between them make up a first SE arm. When a partial rather than a complete spin-flip process occurs, four neutron waves appear in the space after the first flipper. Two of them have flipped spin and therefore a double splitting in $k$, while the two others have nonflipped spin, hence no splitting. As was shown in Refs. [5,6], these waves really coexist and interfere, and each pair of these produces a distinct interference pattern. The question arises if one can use this neutron split into four distinct waves in a neutron scattering experiment, and then, what information one can obtain from it.

A standard spin-echo technique operating with two waves is used to study a pair-correlation function in space or in time. The spin precession, which is equivalent to a splitting of the initial plane wave into two plane waves, accumulates a phase shift until the end of this SE arm. This phase shift corresponds to a distance $R_{1}$ between the fronts of the split waves (or time $t_{1}$ in inelastic processes) $[7,8]$. The neutron thus prepared as a probe passes through the sample to test its properties on a length scale $R_{1}$ (or time scale $t_{1}$ ). The second SE arm collects the two neutron waves to compare their states after the interaction with the sample. In experiment it is more correct to use the concept of the "correlation volume" of the neutron beam described in detail in Ref. [8]. The correlation volume may be intuitively defined as those regions where coherent properties of the neutrons are significant. These properties are described by the correlation function of the as-prepared incoming beam, which is the Fourier transform of its resolution function. Thus, in case of spin echo, the correlation volume is actually split and the spinecho time $\tau_{N S E}$, or the spin-echo length $\delta_{N S E}$, is much longer 
than the correlation time, or the correlation length of the incoming beam. "This splitting of the correlation volumes results in the fact that the spin-echo spectrometer measures the time dependence of the scattering system correlation function directly" [8]. Nevertheless, for the purpose of the clear definition and distinction of the split waves we use through the present paper the terminology of one plane wave being split into two or more others.

As described above, the initial neutron wave may be split into four waves in the first SE arm by setting the spin-flip probability of the first resonant coil equal to $1 / 2$ so that each of the two neutron waves (with opposite spin states) entering the coil is divided into two at the exit. Now, in the precession path of the first SE arm these four neutron waves produce phase shifts corresponding to distances $R_{1}, R_{2}, R_{3}$ (or time intervals $\left.t_{1}, t_{2}, t_{3}\right)$. The resonant coil at the end of the first SE arm stops the precession of all waves in time and in space. The neutron thus prepared probes the sample simultaneously on the length scale $R_{1}, R_{2}, R_{3}$ (or at the same point on the time scale $\left.t_{1}, t_{2}, t_{3}\right)$. The second SE arm mirrors the action of the first one, so at its end the four neutron waves are combined. In this way these four neutron waves after their interaction with the sample contribute to the SE pattern. They allow one to collect information from two correlation distances in real space, time, or both of them and to measure a composite correlation function, which may be of interest in case complex structures are present in the sample. Thus the resonance coil is realized as a basic element for a new type of the interferometer $[9,10]$ and opens a new field, which may be referred to as neutron resonance interferometry. Many basic ideas about phase accumulation due to spin precession are discussed in a recent book by Rauch and Werner [11].

In this paper we concentrate mostly on the development of the technique to deal with four-wave neutron-resonance spin echo. We describe the principles of this spin echo and demonstrate its realization on the present setup, which was built as spin-echo SANS installation [12,13]. In this particular setup we used adiabatic neutron-resonant spin flippers [14-16]. Since a spin-flip process with $\rho=1$ occurs for the "white" neutron spectrum in this flipper, the whole spectrum of neutron wavelengths is involved in the precession, in contrast to experiments where conventional resonance flippers are used. It is also possible to produce four-wave SE pattern with ordinary neutron-resonant spin flippers. Then this pattern is realized for a limited wavelength range only.

To reveal the properties and the principles of the phase compensation process, one must offset the spin-echo setup by means of a so-called compensation coil and measure the polarization of the beam in making a scan with this coil around SE. The polarization signal obtained in this way has a characteristic shape and is called "SE pattern." Depending on whether $\rho=0$ or $\rho=1$, we denote these patterns as "DC-SE pattern" or "RF-SE pattern." The full width at half maximum of this pattern depends on the wavelength spread of the beam, which produces this pattern. Off setting the spin-echo setup is equivalent to scanning the so-called "phase-ofwaves" diagram for the precession modes introduced above.

In principle, two different positions can be chosen for the compensation coil to make this scan in phase-of-waves dia-

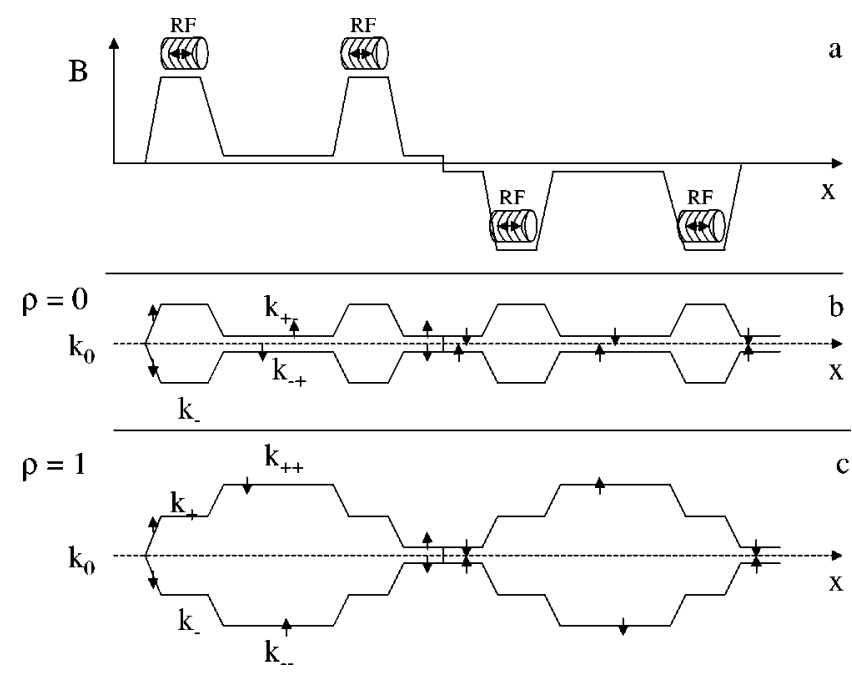

FIG. 1. (a) Sketch of the system of magnetic fields of a neutronresonance spin-echo setup. Each arm consists of two RF spin flippers with a guide field between them. (b) $(k, x)$ diagram of the wave vectors as a function of position along the beam for flippers with spin-flip probability $\rho=0$ and $\vec{P}_{0} \perp \vec{B}_{0}$. (c) $(k, x)$ diagram for $\rho=1$ and $\vec{P}_{0} \perp \vec{B}_{0}$.

gram. The first option is to locate the coil between the two spin flippers of the first SE arm. The second one is to locate it between the SE arms, or upstream or downstream the whole setup, which is equivalent. The compensation coil or field located at different places affects the superposed waves of the RF and DC SE patterns differently. In the paper we show experimentally and theoretically that the combination of the possibilities of the adiabatic NRSF with one of the options of the compensation coil makes it possible to influence these four waves in such a way that they are focused in one single point of the phase-of-waves diagram.

The paper is organized in the following way. The principal scheme of the NRSE with partial spin flip is shown in Sec. II. We give also a receipt for focusing the four-wave SE pattern using the parameters of the NRSF with adiabatic passage. The principal difference of the location of the compensation fields at the installation is also discussed. Section III gives details concerning the setup. The results of the measurements are given in Sec. IV and in Sec. V. Section VI presents a short discussion on the application of the proposed technique to NRSE in connection with the correlation function. The concluding remarks are given in Sec. VII.

\section{NRSE WITH PARTIAL SPIN FLIP $\rho=1 / 2$}

\section{A. Principal scheme}

Figure 1(a) shows schematically the system of the magnetic fields of two RF spin flippers with a guide field between them as a first spin-echo arm and an identical system with negative value of the field as the second SE arm. For the simplest case, when no spin flip occurs $(\rho=0)$, the $(k, x)$ diagram of the wave vectors as a function of position along the beam is shown in Fig. 1(b). At the positions of the flippers, the neutron spin states split by the magnetic field $B_{0}$ 
into waves with wave vectors $k_{-}=k_{0}-\mu_{n} B / \hbar v$ and $k_{+}=k_{0}$ $+\mu_{n} B / \hbar v$. The phase shift, provided by this splitting, is proportional to the area between $k_{+-}$and $k_{-+}$and equals

$$
\phi_{1}=\int\left[k_{+-}(x)-k_{-+}(x)\right] d x,
$$

where the integral is taken over the whole length of the SE arm. $k_{+-}, k_{-+}$indicate the paths of the neutron waves are split in the first flipper. This is referred to as "DC mode" of precession, that is, Larmor precession in the static field of the flippers only. The phase shift between these waves is compensated by an opposite phase shift in the second SE arm. In order to observe this compensation, the initial polarization must be set in the "precession" mode, i.e., perpendicular to the quantization axis: $\vec{P}_{0} \perp \vec{B}_{0}$.

When the spin-flip process is complete $(\rho=1)$, the neutron states exchange a photon of energy $\hbar \omega_{0}=2 \mu_{n} B_{0}$ with the RF field. So during the spin flip in the area of RF field, neutron spin states with $k_{-}$and $k_{+}$will gain or lose an amount of potential energy $\Delta E=2 \mu_{n} B_{0}$. Upon leaving the static field, their potential energy is released as a kinetic energy change. Then the splitting of the wave vector is doubled: $k_{-}$becomes $k_{--}=k_{0}-2 \mu_{n} B_{0} / \hbar v ; k_{+}$becomes $k_{++}=k_{0}+2 \mu_{n} B_{0} / \hbar v$. This is shown in the $(k, x)$ diagram [Fig. 1(c)]. In the zero-field region after the flipper the phase shift in each of them is both $(x, t) \quad$ dependent: $\quad \phi(x, t)=\int_{0}^{x}\left[k_{++}\left(x^{\prime}\right)-k_{--}\left(x^{\prime}\right)\right] d x^{\prime}-2 \omega_{0} t$. This growth of both the spatial and time parts of the phase, i.e., precession, may be halted by transmitting the neutron through another RF spin flipper identical to the first one. By emitting or absorbing the photon in this flipper, both $k_{++}$and $k_{--}$return to $k_{0}$ and also the difference in evolution rate in time $\omega_{0}$ disappears. The amount of this precession (or precession phase) is proportional to the area between $k_{++}(x)$ and $k_{--}(x)$ and is equal to

$$
\phi_{2}=\int\left[k_{++}(x)-k_{--}(x)\right] d x .
$$

This phase shift is compensated by the second SE arm with two RF spin flippers operating at $\rho=1$. This mode of precession is henceforth referred to as "RF mode" of precession. It is worthwhile to notice that such a scheme was proposed for the first time in Refs. [1-3] and was developed later in Refs. $[5,6]$ for the case of an incomplete spin-flip process.

When the spin-flip process is incomplete $(0<\rho<1)$, all four $k$ levels in the space between flippers will be occupied [Fig. 2(a)]. The second flipper doubles the number of waves by splitting them in $(k, \omega)$ space again. Thus, at the end of the first SE arm we have eight waves at four different $k$ levels, indicated as an arrow in Fig. 2(a). These waves interfere with phase shifts $\phi_{1}, \phi_{2}, \phi_{3}$, and $\phi_{3}^{\prime}$, where

$$
\phi_{3}=\int\left[k_{++}(x)-k_{-+}(x)\right] d x
$$

and

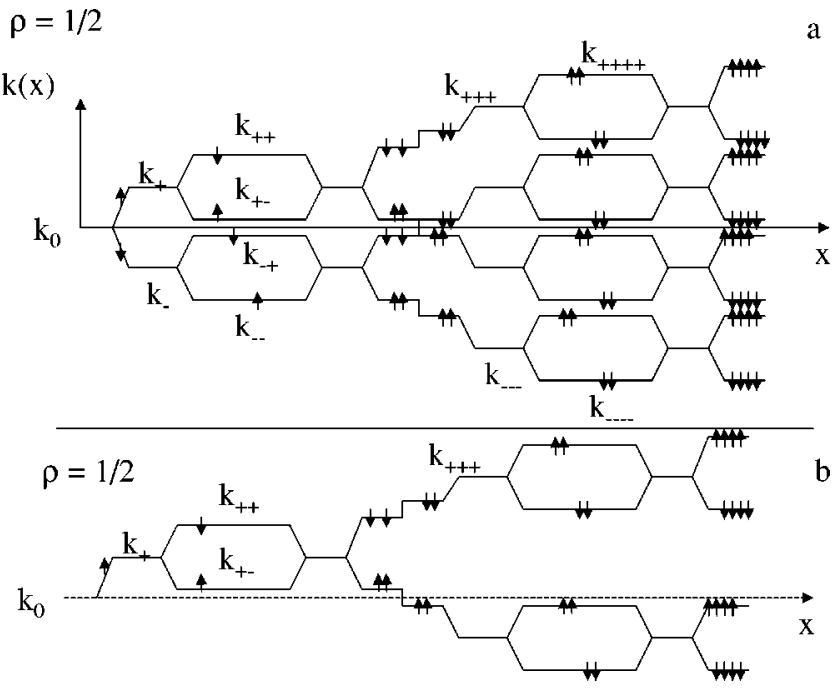

FIG. 2. (a) $(k, x)$ diagram for $\rho=1 / 2$ and $\vec{P}_{0} \perp \vec{B}_{0}$. (b) $(k, x)$ diagram for $\rho=1 / 2$ and $\vec{P}_{0} \| \vec{B}_{0}$.

$$
\phi_{3}^{\prime}=\int\left[k_{+-}(x)-k_{--}(x)\right] d x .
$$

One can show that $\phi_{3}=\phi_{3}^{\prime}=\left(\phi_{1}+\phi_{2}\right) / 2$, so these interference "terms" correspond to half the "sum area" taken for cases $\rho=1$ [Fig. 1(c)] and $\rho=0$ [Fig. 1(b)].

One can simplify the situation by setting the initial polarization parallel to the field: $\vec{P}_{0} \| \vec{B}_{0}$, thus letting a neutron occupy only one $k$ level initially and keep $\rho=1 / 2$ [Fig. 2(b)]. The corresponding phase shift is produced by the $k$ levels $\left(k_{++}\right.$and $\left.k_{+-}\right)$and is equal to

$$
\phi_{4}=\int\left[k_{++}(x)-k_{+-}(x)\right] d x,
$$

which corresponds to half the "difference area" taken for cases $\rho=1$ [Fig. 1(c)] and $\rho=0$ [Fig. 1(b)]: $\phi_{4}=\left(\phi_{2}-\phi_{1}\right) / 2$. This last term represents the interference patterns in Ramsey's problem of the separated oscillating fields $[17,18]$.

From the proposed scheme it is seen that the phases of the four waves created in the first SE arm are compensated by the phase shifts in the second SE arm and interfere with each other.

\section{B. Compensation coils}

SE focusing occurs when the phase shift produced in the first SE $\operatorname{arm} \phi_{\mathrm{I}}$ is equal to that of the second $\operatorname{arm} \phi_{\mathrm{II}}$, so that

$$
\phi_{\mathrm{I}}=\phi_{\mathrm{II}}, \quad \text { where } \quad \phi_{\mathrm{I}, \mathrm{II}}=\int_{0}^{L_{\mathrm{I}, \mathrm{II}}} \Delta k_{\mathrm{I}, \mathrm{II}}(x) d x .
$$

It never happens automatically because of small differences either between $L_{\mathrm{I}}$ and $L_{\mathrm{II}}$ or between $\Delta k_{\mathrm{I}}$ and $\Delta k_{\mathrm{II}}$. For each precession option of the SE machine (DC and RF), a small, but practically unavoidable, distortion between SE arms is present. These values are not necessarily equal. Therefore we need to compensate the value $\phi_{C}=\phi_{\mathrm{I}}-\phi_{\mathrm{II}}$ 

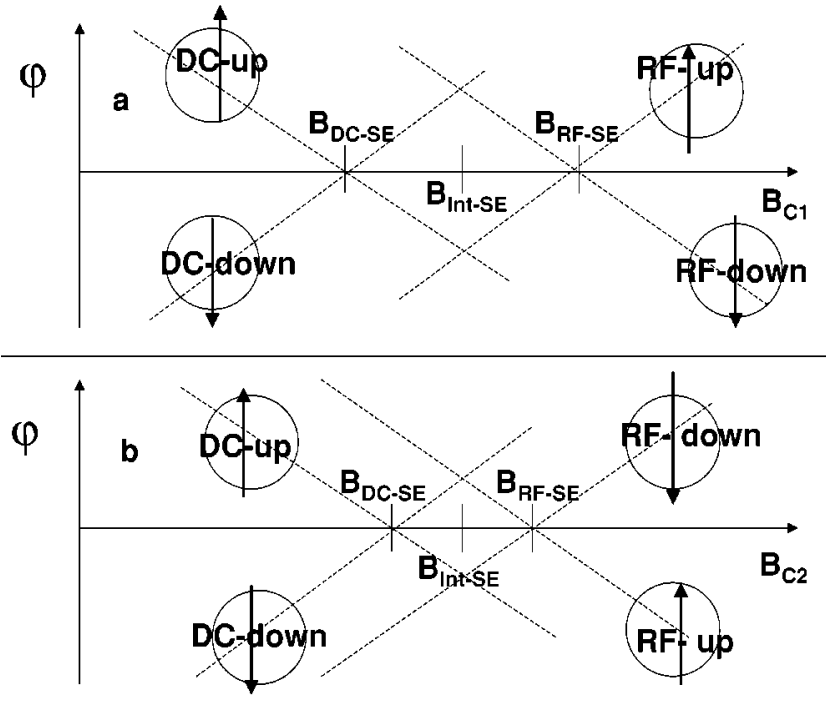

FIG. 3. The phase-of-waves diagram of the four individual waves ( $\mathrm{DC}^{u p}$, $\mathrm{DC}^{\text {down }}, \mathrm{RF}^{u p}$, and $\mathrm{RF}^{\text {down }}$ ) as a function of the magnetic field of the compensation coil located at one of the two different positions: (a) between two spin flippers of the same SE arm, field value denoted $B_{C 1}$; (b) between the two SE arms (upstream or downstream the setup), field value denoted $B_{C 2}$.

using a compensation coil and to do this for $\phi_{C}(\mathrm{DC})$ and for $\phi_{C}(\mathrm{RF})$, successively.

Figures 3 and 4 show schematically the phase-of-waves diagram of the four individual waves as a function of the magnetic fields $B_{C 1}$ and $B_{C 2}$ of the compensation coils $C 1$ and $C 2$, respectively. These compensation coils are located at one of the two different positions: (i) between two spin flippers of the same SE arm, field value denoted $B_{C 1}$; (ii) between the two SE arms (upstream or downstream the setup), field value denoted $B_{C 2}$ (see Fig. 5). The importance of the concept of the correlation volumes should be pointed out here [8]. The spheres in Figs. 3 and 4 demonstrate the correlation volumes of each of the four individual waves. In the

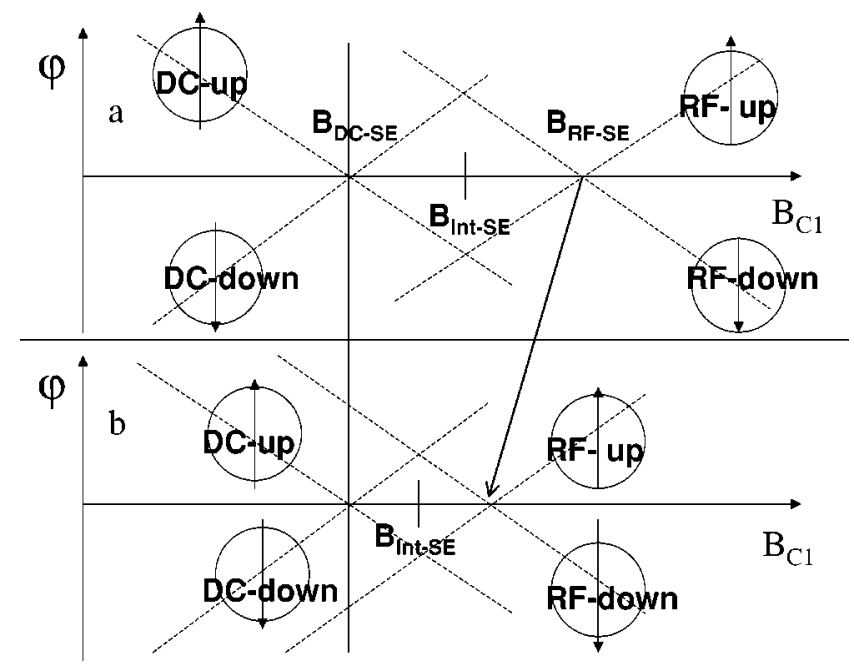

FIG. 4. The phase-of-waves diagram of the four individual waves as a function of the field $B_{C 1}$ for two different values of the gradient field $A$ of the first NRSF.

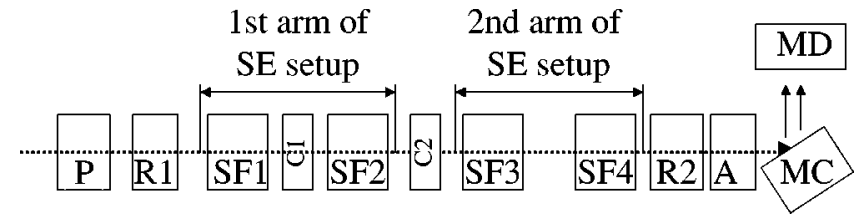

FIG. 5. Schematic drawing of the setup realized at the SP beam line at IRI Delft: $P$, polarizer; $R 1, R 2$, polarization rotators; SF1, $\mathrm{SF} 2, \mathrm{SF} 3$, and SF4 are the NR spin flippers; $C 1$ and $C 2$, the compensating phase coils; A, analyzer; MC, monochromator crystal; $\mathrm{MD}$, detector for quasimonochromatic beam; $\mathrm{D}$, detector for white beam. The system consisting of SF1 and SF2 makes up the first arm of a spin-echo setup while SF3 and SF4 make its second arm. The phase $\phi$ caused by the difference between first and second arms is measured as the field $B_{1}$ generated in $C 1$ and/or $C 2$ needed to compensate this phase.

real experiment the SE pattern arises as soon as the correlation volumes of two different waves are intersected. When no intersection occurs, no SE pattern in observed. Only for the reason of clear distinction for the four different waves we keep using the terminology of the plane split waves.

Suppose we place the compensation coil $C 1$ in the space between RF flippers of the first SE arm. For $\rho=0$ [Fig. 1(b)], an addition of the compensating field increases the phase difference between two neutron waves forming the so-called DC-SE pattern. For $\rho=1$ [Fig. 1(c)], the same addition of field decreases the phase difference between the waves making the so-called RF-SE pattern. Figure 3(a) shows the phase-of-waves diagram as a function of the magnetic field $B_{C 1}$. Each wave is differently affected by the magnetic field $B_{C 1}$ depending on its spin state. An increase of $B_{C 1}$ leads to a decrease of the phase of DC wave with the spin state down (denoted $\mathrm{DC}^{\text {down }}$ ), but to an increase of the phase of rf wave with the same spin state (denoted $\mathrm{RF}^{\text {down }}$ ). It is vice versa for the two waves with spin up [see Fig. 3(a)]. So, we conclude first that the four waves ( $\mathrm{DC}^{\text {down }}, \mathrm{DC}^{u p}, \mathrm{RF}^{\text {down }}$, and $\mathrm{RF}^{u p}$ ) cannot be focused in one single point of the phase-of-waves diagram by using only this compensation coil.

Before proceeding to the other compensation coil $C 2$, consider what an extended scan of $B_{C 1}$ does. An increase of $B_{C 1}$ first makes the two DC waves, $\mathrm{DC}^{u p}$ and $\mathrm{DC}^{\text {down }}$, to coincide at the point called $B_{\mathrm{DC}-\mathrm{SE}}$ [Fig. 3(a)]. Further increase of $B_{C 1}$ separates these two waves, but instead, leads the phases of the wave $\mathrm{DC}^{\text {down }}$ and $\mathrm{RF}^{\text {down }}$ and simultaneously the wave $\mathrm{DC}^{u p}$ with the wave $\mathrm{RF}^{u p}$ to coincide at $B_{\text {Int-SE }}$ [Fig. 3(a)]. Each of these pairs makes different SE patterns, which we call "intermediate" SE. Peculiarly, for both these interference patterns, the spin states of the interfering waves are co directed despite being opposite for both SE patterns. As a consequence, the intermediate SE patterns are only observed when the polarization of the incoming beam is parallel to the quantization axis (along the field $B_{C 1}$ ). At last what happens with even further increase of the field $B_{C 1}$ is focusing the two RF waves $\left(\mathrm{RF}^{u p}\right.$ and $\mathrm{RF}^{\text {down }}$ ) at the point $B_{\text {RF-SE }}$ [Fig. 3(a)]. This RF-SE as well as the DC-SE is observed for the incoming polarization perpendicular to the quantization axis because here two waves with opposite spin state interfere.

When we locate a compensation coil $C 2$ outside the SE arms, for instance, between them (see Fig. 5), the addition of 
the compensating field $B_{C 2}$ increases the phase shift both between the two DC and the two RF waves. Thus using the same terminology, an increase of the field $B_{C 2}$ produces a decrease of the individual phase of both the waves $\mathrm{DC}^{\text {down }}$ and $\mathrm{RF}^{\text {down }}$. Again it is vice versa for the waves with spin state up [Fig. 3(b)]. The scan of $B_{C 2}$ shows also four different SE patterns: one for two DC waves at the point $B_{\mathrm{DC}-\mathrm{SE}}$, two for the intermediate patterns $\left(\mathrm{RF}^{u p}\right.$ and $\left.\mathrm{DC}^{\text {down }}\right)$ and $\left(\mathrm{RF}^{\text {down }}\right.$ and $\mathrm{DC}^{u p}$ ) at the point $B_{\mathrm{Int}-\mathrm{SE}}$, and the fourth one is for the two RF waves at the point $B_{\text {RF-SE }}$ [Fig. 3(b)]. All of them are observed for spin perpendicular to the quantization axis.

Thus we see that the different locations of the compensation coil or field affect the superposed waves of the RF and DC SE patterns differently and one single compensating coil cannot focus four waves in one SE pattern. On the contrary, use of two compensation coils, one between the flippers of the first arm and another one between two arms, enables us to focus simultaneously both DC and RF modes.

\section{NRSE focusing with adiabatic spin flipper}

NRSF with adiabatic passage in both SE arms, apart from the possibility to make partial flip (important for multiwave splitting) offer an opportunity to shift the DC and RF patterns independently from the compensation coils $C 1$ and $C 2$.

For an adiabatic passage of the neutron spin one needs $[12,16]$ a permanent homogeneous field $B_{0}$ with added to it a gradient along the neutron path $x=[0, l]$, so that the resonant point $\left(2 \pi \nu_{\mathrm{rf}}=\gamma B_{0}\right)$ occurs approximately at the center of the flipper: $x=l / 2$, where $l$ is the length of the flipper and $\nu_{\mathrm{rf}}$ is the frequency of the oscillating field. We describe the gradient field as $B(x)=A^{\prime} \cos (\pi x / l)$. Second, one needs an oscillating field $B_{\mathrm{rf}}(x, t)$ which increases from the entrance to the middle, and then decreases toward the exit of the flipper, described by $B_{\mathrm{rf}}(x)=A^{\prime \prime} \sin (\pi x / l) \cos \left(\omega_{0} t\right)$.

The exact expression for the spin-flip probability, derived in Refs. $[12,16]$, is given as

$$
\rho=1-\sin ^{2} \phi /\left(k^{2}+1\right),
$$

where $\phi$ is the phase of the spin in the magnetic field of the reference frame rotating at the frequency $\omega_{\mathrm{RF}}$ and $k=\omega_{L} / \Omega$ is the adiabaticity parameter of the reference frame. It is clear that the spin flip is complete for all neutrons with a velocity (wavelength) satisfying the adiabatic condition. This means the adiabaticity parameter $k$ obeys the inequality $k=\gamma_{n} A l / \pi v \gg 1$. The spin-flip probability may be readily changed between 1 and 0 by varying the adiabaticity parameter $k$ from $k \gg 1$ to $k=0$ in one single point of the flipper. It is most convenient to arrange this by changing the amplitude of the RF field $A^{\prime \prime}$ from $A_{\max }^{\prime \prime}$ to 0 . When the spin-flip process is partial, the neutron state becomes a coherent sum of the states $\left(\mathrm{RF}^{u p}, \mathrm{RF}^{\text {down }}, \mathrm{DC}^{u p}\right.$, and $\mathrm{DC}^{\text {down }}$ ). During passage through the flipper the phase shifts of the flipped (RF) states and nonflipped (DC) states are different but nevertheless certain. Thus the phase of the flipped states is the sum of the contributions coming from the gradient field and RF field. At the same time the unflipped states are affected by neither gradient field nor
RF field, and are influenced by the permanent field $B_{0}$ only.

As was shown in Ref. [12], when the amplitudes of the gradient field and the RF field are equal, $A^{\prime}=A^{\prime \prime}=A$, the precession phase difference $\Delta \Phi$ of the flipped state in the magnetic-field configuration of NRSF with adiabatic passage is

$$
\Delta \Phi_{f}=\omega_{0} \tau+( \pm) \varphi_{f}=\omega_{0} \tau+( \pm)\left(\pi \sqrt{k^{2}+1}\right) .
$$

The first term is the contribution of the rotating frame $\left(\omega_{0} \tau\right)$, as in a conventional flipper [1-3]. The second term $\left(\varphi_{f}\right.$ $\left.=\pi \sqrt{k^{2}+1}\right)$ is the precession phase in the rotating frame. Its sign is determined by the sign of the gradient field $A^{\prime}$ with respect to the spin. In practice, the variation of the effective field in the rotating frame determines the stability of the total phase. Therefore, it is more realistic to replace the phase $\varphi_{f}$ of Eq. (8) by $\int_{0}^{l}\left|B_{e f f}\right| d x$. Then under the adiabatic approximation we can rewrite it in the following way:

$$
\varphi_{f} \approx \frac{\gamma}{v} \int\left|B_{e f f}(x)\right| d x=\frac{\gamma}{v} \int \sqrt{B_{z, e f f}^{2}(x)+B_{x, e f f}^{2}(x)} d x,
$$

where $\quad B_{z, e f f}(x)=B_{0}-\omega_{0} / \gamma+A^{\prime} \cos (\pi x / l)$ and $B_{x, e f f}(x)$ $=A^{\prime \prime} \sin (\pi x / l)$. $A^{\prime}$ and $A^{\prime \prime}$ are the amplitudes of the gradient field and the oscillating field as set by the experimentalist.

Contrary to it, the precession phase $\Delta \Phi_{n}$ of the nonflipped states in the magnetic-field configuration of NRSF with adiabatic passage is

$$
\Delta \Phi_{n}=\int_{0}^{l} B_{0}(x) d x=\int_{0}^{l} B_{0}+A \cos (\pi x / l) d x=B_{0} l .
$$

Hence the SE signal of the nonflipped states does not shift with a change of the gradient.

Thus three parameters of the flipper may be varied: $A^{\prime}$, $A^{\prime \prime}$, and $B_{0}$. The amplitude of RF field varies the spin-flip probability $\rho$ or relative weight of the flipped and nonflipped states. The value of the gradient $A^{\prime}$ changes the phase shift of the flipped states ( $\mathrm{RF}^{u p}$ and $\mathrm{RF}^{\text {down }}$ of Fig. 4) and has no effect on the nonflipped states (DC ${ }^{u p}$ and $\mathrm{DC}^{\text {down }}$ of Fig. 4). Contrary to it, the value of the permanent field $B_{0}$ changes the phase of the nonflipped states and has relatively small influence on the flipped states.

The combination of the effects of the compensation coil and the adiabatic spin flipper is shown in the phase-of-waves diagram in Fig. 4. As discussed in Sec. II B, the scan of the compensation field $B_{C 1}$ produces the SE patterns: DC pattern, intermediate pattern, and RF pattern. A change of the gradient in one of the adiabatic NRSE flippers results in a shift of the position of the RF-SE pattern, as is demonstrated in Fig. 4. In this way all waves may be focused at one point of the phase-of-waves diagram.

\section{LAYOUT OF THE NRSE EXPERIMENT}

The measurements below were carried out using the polarizing mirror setup SP at IRI, Delft, shown schematically in 
Fig. 5. A polychromatic neutron beam emerging from a 2 MW swimming-pool type reactor is polarized by polarizer $P$. Using rotator $(R 1)$ the polarization of the beam is rotated towards the $y$ axis, i.e., perpendicular to the guide field. The first spin-echo arm consisting of two RF spin flippers is located downstream from this rotator $R 1$. In our experiments we set $B_{0}$ equal to $414 \mathrm{G}$ and the frequency of the oscillating field equal to $1.22 \mathrm{MHz}$, respectively, to fulfill the resonance condition at the center of the flipper. The length of the RF coils is $0.1 \mathrm{~m}$ and they are placed at a center-to-center distance of $L=0.9 \mathrm{~m}$. The permanent magnetic field is produced by an electromagnet with pole shoes such that the magnetic field is relatively homogeneous over the length of the flipper. It drops outside both sides of the magnet. Two specially constructed coils were placed between the pole shoes to create the gradient of the permanent field. These coils were wound in such a way that their magnetic field is positive over one half of the magnet and negative over the other half, and having a zero field at the middle. The current passing through these coils is proportional to the magnitude of the magnetic field ( $z$ component) in the rotating frame, denoted $A^{\prime}$ in Sec. II. The RF coil with a diameter of $30 \mathrm{~mm}$ is located in the center between these two coils, with the oscillating field along the $x$ axis. With this construction we could vary the following parameters of the flippers independently: the magnetic field $B_{0}$ from 0 to $1000 \mathrm{G}$, the gradient $2 A^{\prime}$ of the permanent field from 0 to $80 \mathrm{G}$, and the amplitude of the oscillating field from 0 to $40 \mathrm{G}$. After transmission through the two RF flippers, neutrons pass the field stepper. The second arm consists of two other identical flippers. Their static magnetic field $\vec{B}$ is opposite to the static magnetic field in the first two flippers. Two compensation coils $C 1$ and $C 2$ of length $0.40 \mathrm{~m}$ were located at some position between the two RF flippers in the first SE arm and between SE arms, respectively. Both coils can produce a static magnetic field $B_{1}$ in the range from -80 to $+80 \mathrm{G}$. The second arm of the SE setup is followed by a second polarization rotator $(R 2)$ and an analyzer (A). By Bragg reflection at a monochromator crystal (MC), neutrons with wavelength $\lambda$ $=0.19, \ldots, 0.23 \mathrm{~nm}$ are reflected into various detectors of the multidetector (MD). The spread of the wavelength spectrum in each detector is $0.02 \mathrm{~nm}$, approximately. The polarization was measured as a function of the precession phase $\phi$ acquired after passing through both SE arms. The latter is varied in different ways by the values of the magnetic fields $B_{C 1}$ and $B_{C 2}$ in the phase compensation coils $C 1$ and $C 2$ and by the parameters of the adiabatic flippers $A$ and $B_{0}$.

\section{RESULTS: NRSF WITH ADIABATIC PASSAGE}

\section{A. Spin-flip probability}

The spin-flip probability $\rho$ is an important quantity which determines the amplitude of the SE signals. It was measured in a separate experiment with only one flipper between polarizer and analyzer as a function of the permanent field $B_{0}$ while the gradient coil was set to a constant value. In this setup one measures in fact $P_{z}$. From the definition of the polarization, $\rho$ is connected to $P_{z}$ by
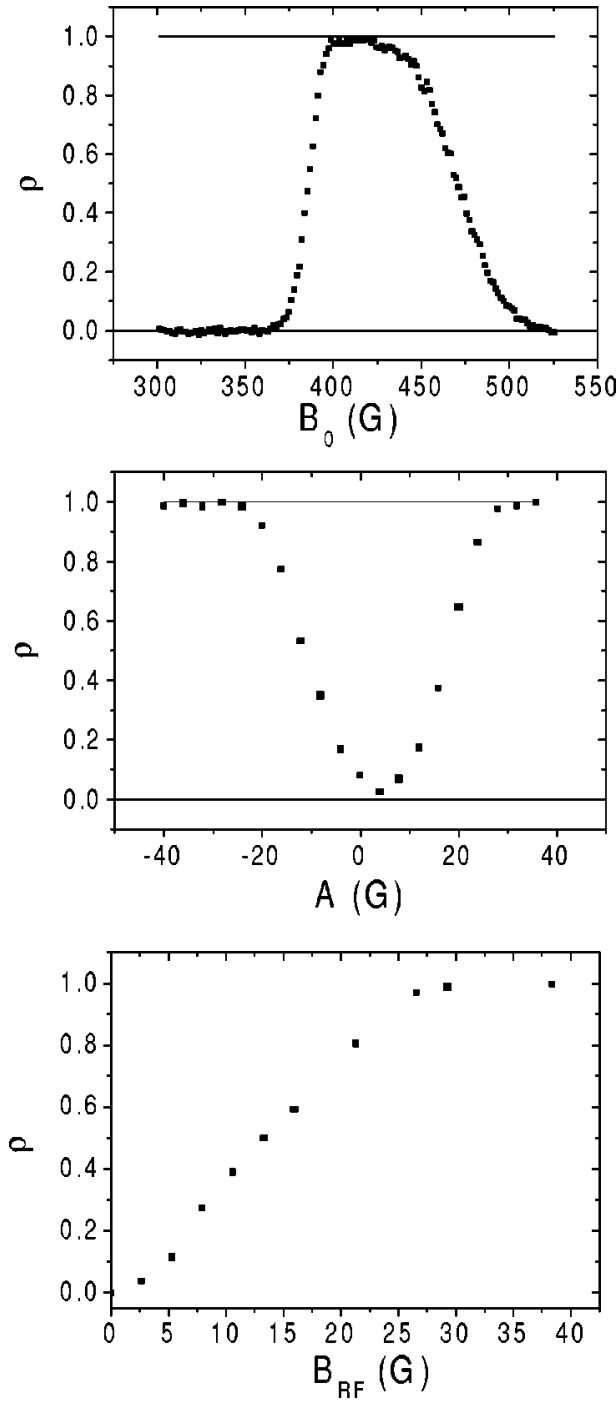

FIG. 6. Dependence of the spin-flip probability $\rho$ in one NR spin flipper on the permanent magnetic field $B_{0}(\mathrm{a})$; on the gradient field $A$ (b); on the oscillating field value $B_{\mathrm{RF}}$ (c) for wavelength $\lambda$ $=0.2 \mathrm{~nm}$.

$$
\rho=\left(1-P_{z}\right) / 2 .
$$

Figure 6(a) shows a broad resonance line measured with $\nu$ equal to the resonance frequency $\nu=1.22 \mathrm{MHz}\left(B_{0}^{\text {res }}\right.$ $=414 \mathrm{G})$. The width of the resonance line at half height $(\rho$ $=1 / 2$ ) is determined by the magnitude of the gradient $2 A^{\prime}$ of the permanent magnetic field. In our case the gradient comprises $80 \mathrm{G}$ from minimum to maximum as can be deduced from the width of the curves at their bottom. The field range with $\rho \approx 1$, a so-called "plateau," extends over a range of about $40 \mathrm{G}$ around $B_{0}^{\text {res }}$, which is about half the field gradient. The magnetic field therefore may be changed within the range $B_{0}^{\text {res }}-A<B_{0}<B_{0}^{\text {res }}+A$. This change in field $B_{0}$ does not affect the probability but significantly changes the phase of the DC mode while the phase of the RF mode stays unchanged. The asymmetry of the resonance line is connected with the nonsharp boundary of the magnetic field $B_{0}$.

Figure 6(b) shows the dependence of the spin-flip probability $\rho$ on the value of the gradient $A^{\prime}$ at optimal $B_{0}$ 
$=414 \mathrm{G}$ and the maximal value of $B_{\mathrm{RF}}=38 \mathrm{G}$. The value of the gradient was varied from -40 to $+40 \mathrm{G}$ in the experiment. For sufficiently large absolute value $(25 \mathrm{G})$ of the gradient $|A|$, the spin-flip process becomes complete $\rho \approx 1$. This allows us to change the value of the gradient beyond $25 \mathrm{G}$ in order to change the phase of the SE-RF mode. As was shown above, the position of the SE-DC pattern does not change with the gradient $A$.

Figure 6(c) shows the dependence of the spin-flip probability $\rho$ on the value of the $B_{\mathrm{RF}}$ at optimal $B_{0}=414 \mathrm{G}$ and the maximal value of the gradient $A=40 \mathrm{G}$. We used this dependence for setting $\rho=1 / 2$ to provide the four-wave splitting in this SE experiment. It was verified also that the position of the SE does not significantly change with the value of the $B_{\mathrm{RF}}$.

\section{B. Phase shift by NR spin flipper with gradient}

We find it more convenient to vary the gradient of one neutron-resonance spin flipper in focusing the four-wave SE, since a change in the parameter $B_{0}$ makes, although insignificantly, some shift of the RF-SE pattern along with a strong shift of the DC-SE pattern. The value of the gradient $A$ has no effect on the DC-SE pattern at all while its effect on the RF-SE pattern is significant. To demonstrate this we show in Fig. 7 the position of the SE-RF pattern as a function of the field $B_{C 1}$ of $C 1$. $C 1$ was operated only over the relevant range to reveal the SE patterns. As was mentioned above, the NRSF contributes to the phase shift of the neutron passing through it as $\pm \varphi_{f}$ [Eq. (10)], where the sign ( \pm ) is determined by the mutual orientation of the spinor and gradient of the field with respect to the neutron velocity. Thus, when the signs of all four flippers are positive $(++++)$, the combination of the contributions to the total phase shift is $\left[\left(+\varphi_{f 1}\right.\right.$ $\left.\left.-\varphi_{f 2}\right)-\left(+\varphi_{f 3}-\varphi_{f 4}\right)\right]$, because the first flipper changes the sign of spinor and therefore it will be opposite for the second flipper. Thus, for all gradients $(++++)$ positive the phase shifts induced by all flippers to the RF-SE mutually cancel; it is also true for all gradients (----) negative. When one of the gradients changes sign (say, the first one), the cancellation does not hold and the SE pattern is shifted. Considering the contributions of the gradient fields of all flippers as identical $\varphi_{f 1, f 2, f 3, f 4}=\varphi_{G R}$, we can change the total phase shift $\varphi_{f, t o t}$ of the RF-SE pattern from $-4 \varphi_{G R}$ to $+4 \varphi_{G R}$. This is shown in Fig. 7(a) for the (+--+) combination with $\varphi_{f, t o t}$ $=4 \varphi_{G R}$; in Fig. 7(b) for the (---+) combination with $\varphi_{f, t o t}$ $=2 \varphi_{G R}$; in Fig. 7(c) for the (----) and $(++++)$ combinations with $\varphi_{f, t o t}=0$; in Fig. 7(d) for the $(+++-)$ combination with $\varphi_{f, t o t}=-2 \varphi_{G R}$; and in Fig. 7(e) for the $(-++-)$ combination with $\varphi_{f, t o t}=-4 \varphi_{G R}$. As was mentioned above, the DC echo is not influenced by the gradients $A$ at all. Its location also is shown in Fig. 7(f). It coincides practically (but not exactly) with the RF-SE with gradients $(++++)$ and $(----)$.

\section{RESULTS: FOUR-WAVE SE FOCUSING}

The four neutron waves generated in the first SE arm of the NRSE experiment may be focused into one point of the

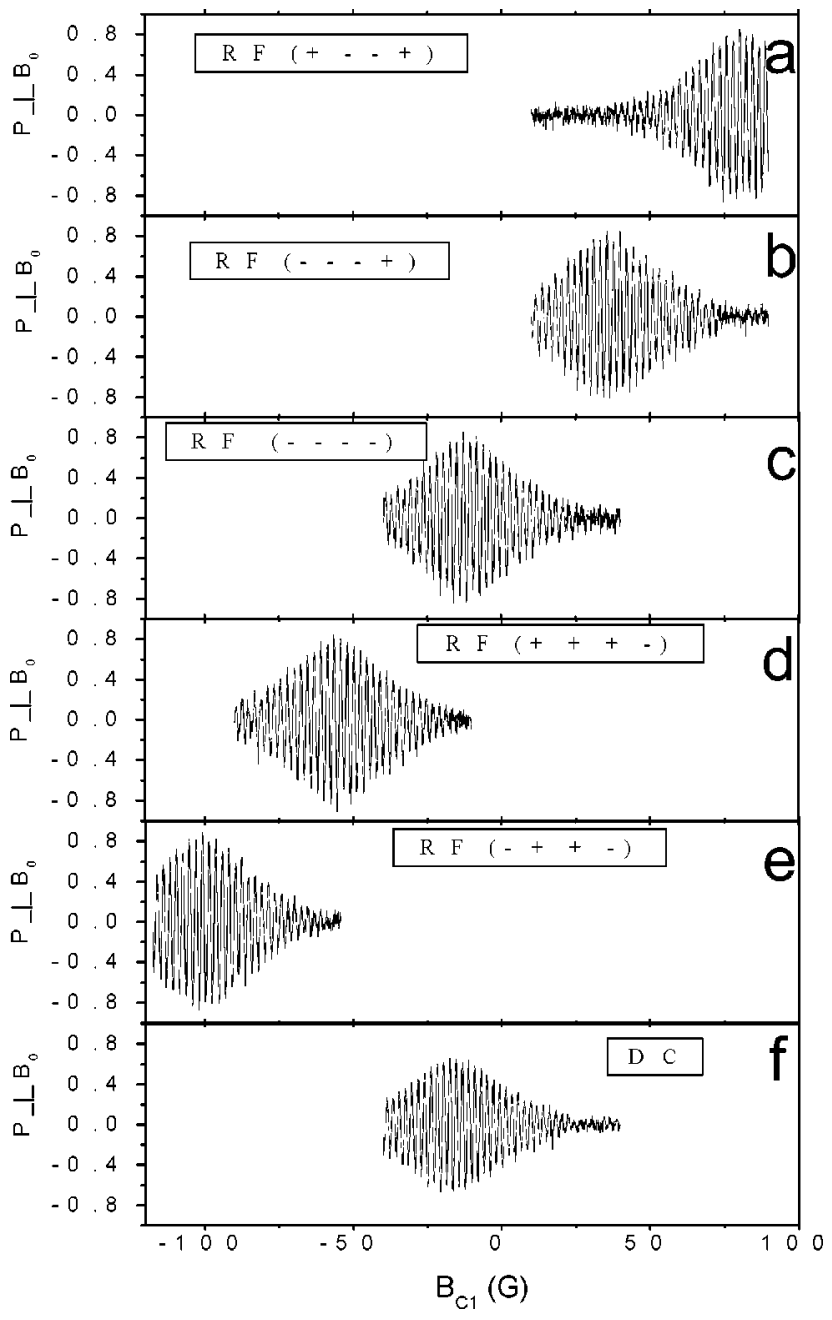

FIG. 7. Spin-echo spectra as a function of $B_{C 1}$ for five different combinations of the signs of the field gradients in flippers (a)-(e). The DC-SE position is the same for all combinations (f).

phase-of-waves diagram. To produce such focusing one needs at least two independent tools to influence these waves. In Sec. I we denoted them as $\mathrm{RF}^{u p}, \mathrm{RF}^{d o w n}, \mathrm{DC}^{u p}$, and $\mathrm{DC}^{\text {down}}$; these waves compose the RF-SE and DC-SE patterns, respectively. The independence of the tools means not only that they are energized from different power supplies but that the magnetic fields, provided by these tools, affect the RF waves and DC waves differently as was explained in Sec. II B. In accordance with this definition, we can consider action of $C 1$ and action of the gradient field of the adiabatic NRSF as independent. The same applies to actions of $C 1$ and $C 2$ and for $C 2$ and the gradient field. These three possible combinations are now of particular interest for us.

\section{A. Focusing with $C 1$ and the gradient field of NRSF}

If we do not take special precautions, the scan made by the field of $C 1$ reveals the RF-SE pattern $(\rho=1)$ at a certain point. A scan made at $\rho=0$ with the same phase coil reveals the DC-SE pattern at a point far away from the RF-SE pattern. In correspondence to the scheme in Fig. 3(a), we focus 

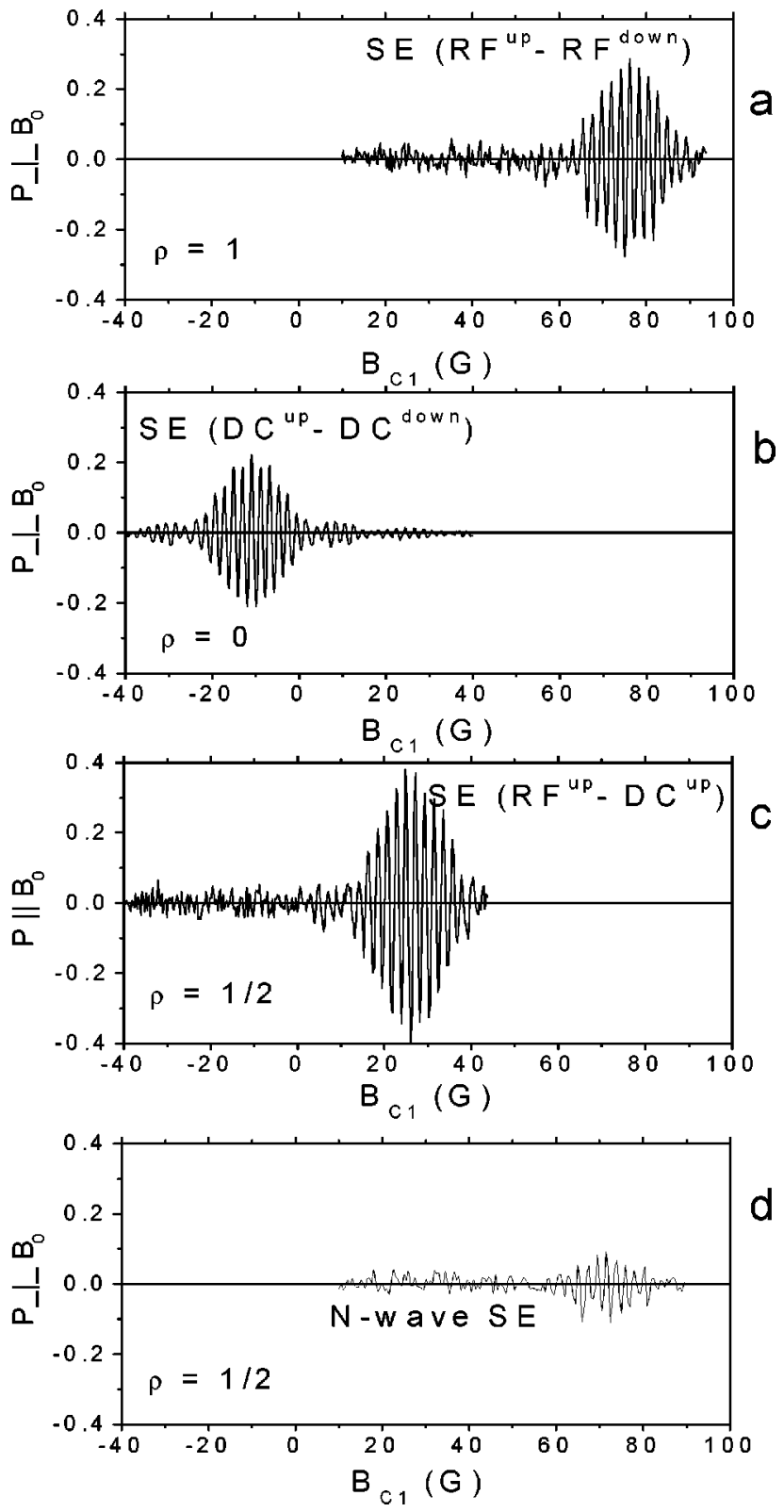

FIG. 8. The polarization as a function of the $B_{C 1}$ for the component perpendicular to the quantization axis: $\rho=1$ (a), $\rho=0$ (b), $\rho=1 / 2$ (c), and the component parallel to the quantization axis $\rho$ $=1 / 2$ (d). Four waves $\left(\mathrm{DC}^{u p}, \mathrm{DC}^{\text {down }}, \mathrm{RF}^{u p}\right.$, and $\left.\mathrm{RF}^{\text {down }}\right)$ do not come in SE in one point.

the two RF waves at $B_{C 1}=76 \mathrm{G}$ and the two DC waves at $B_{C 1}=-11 \mathrm{G}$ [Fig. 8(a) and 8(b), respectively]. When we set $\rho=1 / 2$ in all flippers, we can focus the waves $\mathrm{RF}^{u p}$ and $\mathrm{DC}^{u p}$ as well as the $\mathrm{RF}^{\text {down }}$ and $\mathrm{DC}^{\text {down }}$ at the point $B_{C 1}=33 \mathrm{G}$ [Fig. 8(c)]. We denoted this SE pattern as intermediate (Int). There are two features of the Int-SE pattern: (i) it can be observed only when polarization is parallel to the quantization axis (along the field); (ii) it is halfway between $B_{\mathrm{RF}-\mathrm{SE}}$ and $B_{\mathrm{DC}-\mathrm{SE}}$.

Thus we indeed can identify the four different waves contributing to these SE patterns. Since all four waves are not in focus simultaneously, no SE pattern can be observed in the polarization components perpendicular to the quantization
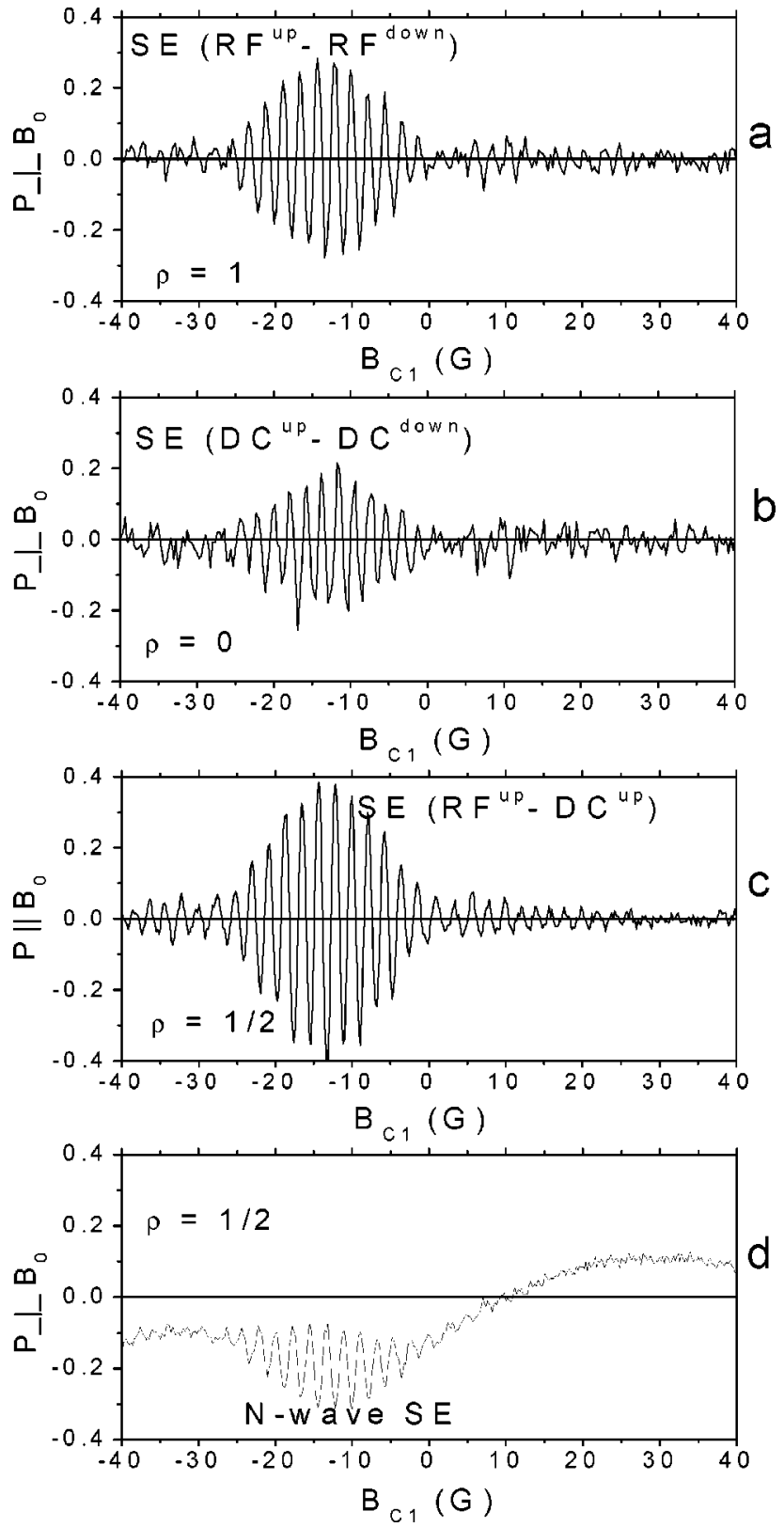

FIG. 9. The polarization as a function of the $B_{C 1}$ for the component perpendicular to the quantization axis: $\rho=1$ (a), $\rho=0$ (b), $\rho=1 / 2$ (c), and the component parallel to the quantization axis $\rho$ $=1 / 2(\mathrm{~d})$.

axis, when $\rho=1 / 2$. Absence of a four-wave SE pattern at the intermediate position is also demonstrated in the experiment, see Fig. 8(d).

In order to focus all four waves at the same point of the $B_{C 1}$, we change the value of the gradient $A$ such that the position of the RF-SE pattern shifts toward the position of DC-SE pattern. This is shown in Fig. 9. We have measured the polarization of the beam perpendicular to the quantization axis for three different cases: $\rho=1$ [Fig. 9(a)], $\rho=0$ [Fig. 9(b), and $\rho=1 / 2$ [Fig. 9(c)]. Additionally, the polarization parallel to the quantization axis was measured for $\rho=1 / 2$ [Fig. 9(d)]. Thus when all four waves are focused in one point of the phase-of-waves diagram, then four-waves SE is 


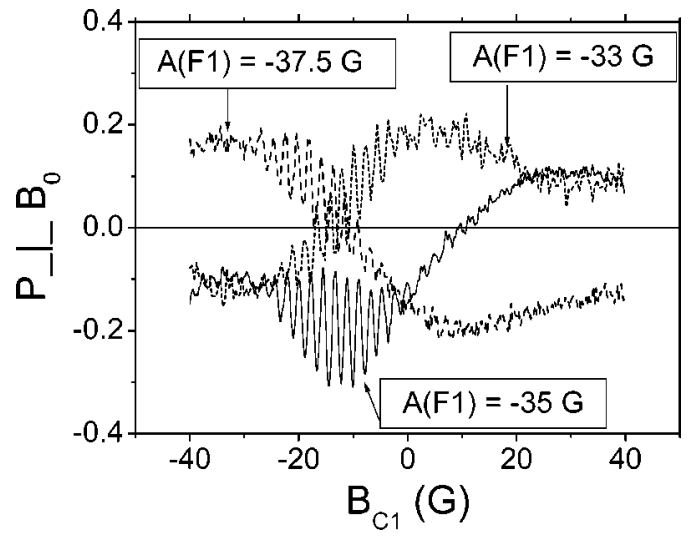

FIG. 10. The polarization as a function of the $B_{C 1}$ for the component perpendicular to the quantization axis $\rho=1 / 2$ at different values of the gradient field $A=33,35,37.5 \mathrm{G}$.

implicitly observed at the point of focusing for $\rho=1 / 2$ and $P_{0} \perp B_{0}$.

It is interesting to point out that the polarization perpendicular to the quantization axis $P_{\perp}$ is not 0 far out of $\mathrm{SE}$ position but shows a slow waving behavior as a function $B_{C 1}$. This is connected with the positions of the two DC and two RF waves on the phase-of-waves diagram. It is well seen in Fig. 4(b) that when the $\mathrm{RF}^{u p}$ and $\mathrm{RF}^{\text {down }}$ waves are out of the RF-SE position, this is also valid for the $\mathrm{DC}^{u p}$ and $\mathrm{DC}^{\text {down }}$ waves. Their added interference patterns result in zero polarization. On the other hand, interference between the $\mathrm{RF}^{u p}$ and $\mathrm{DC}^{\text {down }}$ waves as well as between the $\mathrm{RF}^{\text {down }}$ and $\mathrm{DC}^{u p}$ waves gives polarization $P_{\perp} \neq 0$ independently of the value of $B_{C 1}$. This happens due to the fact that the $\mathrm{RF}^{u p}$ and $\mathrm{DC}^{\text {down }}$ waves (as well as the $\mathrm{RF}^{\text {down }}$ and $\mathrm{DC}^{u p}$ waves) have an equivalent positions on the $B_{C 1}$ scale and their phases change equally as a function of $B_{C 1}$.

However from this consideration there does not follow any slow waving behavior of this polarization component. This fact is connected with difference in effect of the magnetic field $B_{C 1}$ on the DC and RF waves. In the first (linear) approximation, taking into account [Eq. (1)], the magnetic field affects both DC and RF waves equally, but accounting for the small quadratic term, which is different for DC and $\mathrm{RF}$ waves, one can come to the conclusion that it leads to such a smooth waving behavior, as was observed in the experiment. To demonstrate it clearly we show the four-wave SE pattern at three different values of the gradient $A$ of one flipper (Fig. 10). It is seen that the change of the RF phase for $\pi / 4$ makes both smooth (long) wave and quick (short) wave shifted to the same value of $\pi / 4$. The shift of the RF pattern for $\pi / 2$ makes destructive interference and SE vanishes. Such a behavior of the SE pattern in response to $B_{C 1}$ is not convenient for the experimentalist, because it is difficult to predict a polarization value of the intermediate SE pattern and have a maximum of all SE patterns at one point.

\section{B. Focusing with $C 1$ and $C 2$}

One can use another combination of independent tools, which is more convenient for focusing of all four waves:
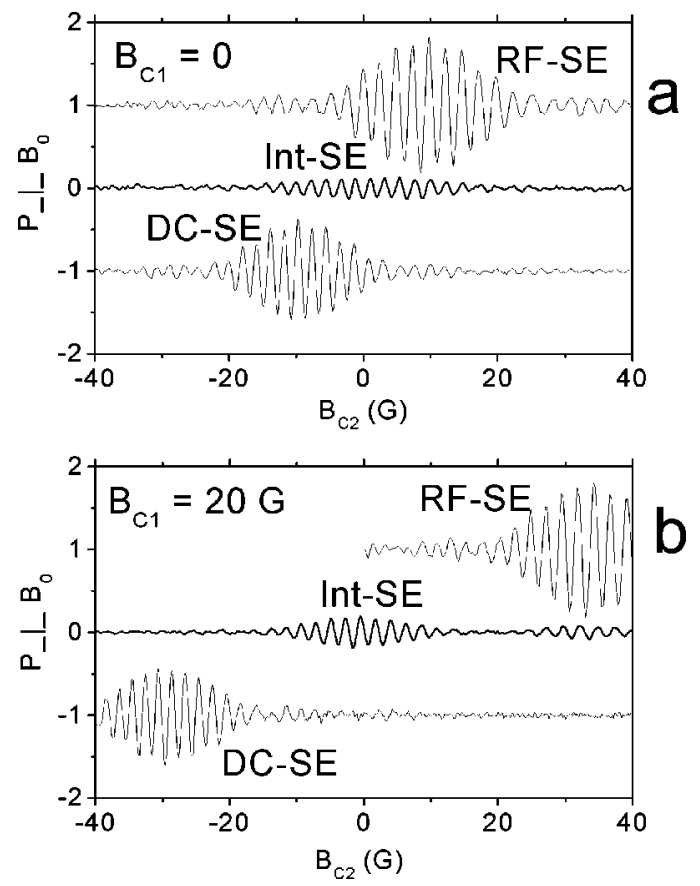

FIG. 11. The polarization as a function of the $B_{C 2}$ for the component perpendicular to the quantization axis: $\rho=1, \rho=0$, and $\rho$ $=1 / 2$ at two different field values of the $C 1 B_{C 1}=0$ (a) and $B_{C 1}$ $=20 \mathrm{G}(\mathrm{b})$.

$\mathrm{RF}^{u p}, \mathrm{RF}^{\text {down }}, \mathrm{DC}^{u p}$, and $\mathrm{DC}^{\text {down}}$. Thus we use the action of $C 1$ to set mutual positions of the RF-SE pattern and the DC-SE pattern, while $C 2$ reveals the SE patterns on its own scale of $B_{C 2}$.

At the arbitrary value of the field of $C 1$, the four waves are out of echo and are able to create separated patterns only. To demonstrate this we measured the polarization as a function of the $B_{C 2}$ for the component perpendicular to the quantization axis at $\rho=1, \rho=0$, and $\rho=1 / 2$ at different field values of the $B_{C 1}$. The observed SE patterns for two particular values of the field of $B_{C 1}$ are shown in Fig. 11: $B_{C 1}=0$ (a) and $B_{C 1}=20 \mathrm{G}$ (b). In this case, in correspondence to the scheme of Fig. 3(b), we focus the two RF waves at $B_{C 2}$ $=10 \mathrm{G}$ and the two DC waves at $B_{C 2}=-10 \mathrm{G}$. In full accord with the scheme of Fig. 3(a), one also can focus intermediate SE patterns built by the $\mathrm{RF}^{u p}$ and $\mathrm{DC}^{\text {down }}$ waves along with the $\mathrm{RF}^{\text {down }}$ and $\mathrm{DC}^{u p}$ waves, at the point $B_{C 2}=0 \mathrm{G}$. To observe the last pattern, we have to create these waves, i.e., to set $\rho=1 / 2$ in all flippers. It is clear that now it can be observed only when the polarization is perpendicular to the quantization axis. The point of the intermediate SE obeys the equation $B_{\mathrm{Int}-\mathrm{SE}}=\left(B_{\mathrm{RF}-\mathrm{SE}}+B_{\mathrm{DC}-\mathrm{SE}}\right) / 2$.

For the case of $B_{C 1}=20 \mathrm{G}$, the picture of the locations of $\mathrm{SE}$ patterns is qualitatively the same. The setting $B_{C 1}$ different from 0 shifts $\mathrm{DC}$ and RF patterns in different directions on the scale $B_{C 2}$ in accord with the statement that $C 1$ and $C 2$ affect the RF and DC waves differently. Thus now we observe RF-SE focus at $B_{C_{2}}=34 \mathrm{G}$ and DC-SE focus at $B_{C_{2}}=$ $-30 \mathrm{G}$, while the focus of the Int-SE pattern is found at the point $B_{C 2}=-2 \mathrm{G}$.

To show explicitly the position of the SE patterns as a function of $B_{C 1, C 2}$, both compensating coils, we determined 


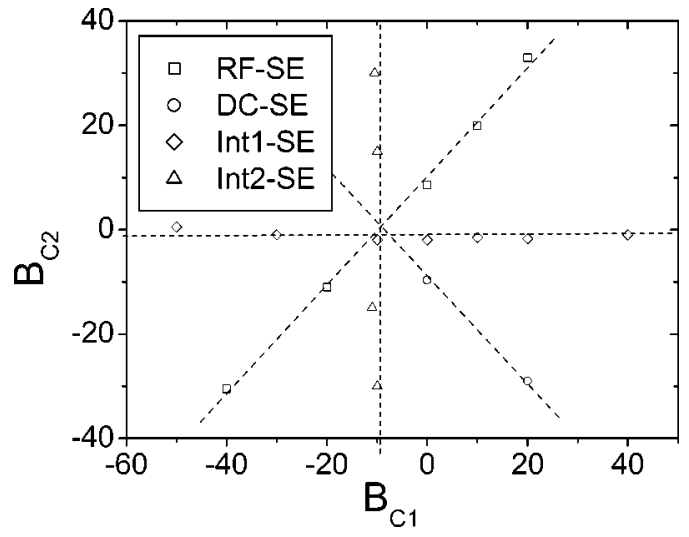

FIG. 12. The position of the SE on the map made of the compensating fields $B_{C 1}$ and $B_{C 2}$. The cross point of two lines for DC-SE patterns and for RF-SE patterns gives the four-wave SE point which is $B_{C 1}=-10 \mathrm{G}$ and $B_{C 2}=0 \mathrm{G}$.

the position of the RF, DC, and Int SE patterns and different values of $B_{C 1}$ as a function of $B_{C 2}$ and vice versa. We can plot a map of SE positions with the compensating fields $B_{C 1}$ and $B_{C 2}$ as axes (Fig. 12). It is well seen in this map that the Int-SE position almost does not change as a function of $B_{C 1}$ or as a function of $B_{C 2}$. We can also find the intersection point of the two lines for the DC-SE patterns and for the RF-SE patterns: $B_{C 1}=-10 \mathrm{G}$ and $B_{C 2}=0 \mathrm{G}$. It corresponds to the point where the four waves $\mathrm{DC}^{u p}, \mathrm{DC}^{\text {down}}, \mathrm{RF}^{u p}$, and $\mathrm{RF}^{\text {down }}$ come in focus: the four-wave SE point. At this point we performed the measurement of the polarization as a function of $B_{C 2}$ with the polarization perpendicular to the quantization axis, and $\rho=1 / 2$ and at $B_{C 1}=-10 \mathrm{G}$ (Fig. 13). It should be noticed that now one can use the gradient of the resonant flipper $A$ to adjust SE such that it would have maximum possible amplitude.

\section{NRSE AND CORRELATION FUNCTION}

The conventional spin-echo technique is used to study a density-density correlation function $G\left(R, \tau_{N S E}\right)$ [7]. The neutron wave splits into two waves and both go the same path

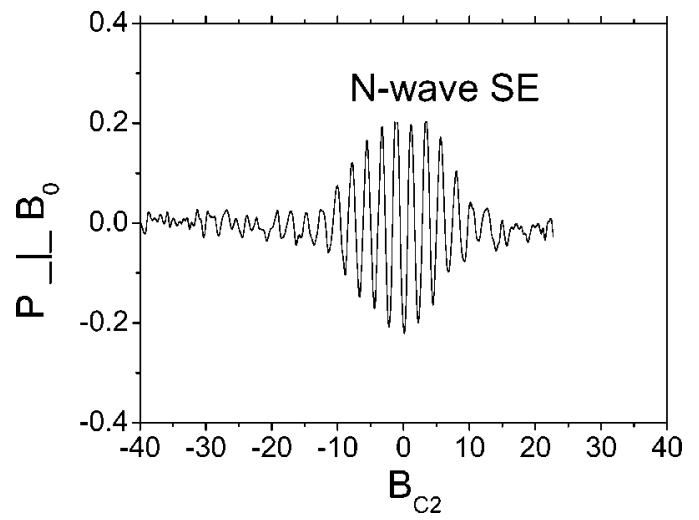

FIG. 13. The polarization as a function of the $B_{C 2}$ for the component perpendicular to the quantization axis $\rho=1 / 2$ at $B_{C 1}=$ $-10 \mathrm{G}$. with a time delay $\tau_{N S E}$ at the sample position, i.e., being split longitudinally. More precisely, the measured quantity

$$
\left\langle\sigma_{x}\right\rangle=\int d \omega S(q, \omega) \cos \left(\omega \tau_{N S E}\right) \sim I\left(q, \tau_{N S E}\right),
$$

where $S(q, \omega)$ is the scattering function and $I\left(q, \tau_{N S E}\right)$ $=\int\left\langle\rho\left(r_{i}, t_{i}\right) \rho\left(r_{i}+R, t_{i}+\tau_{N S E}\right)\right\rangle \exp (i q R) d R$ is the intermediate scattering function [7].

A recent development of the SE technique with tilted fields resulted in a new type of SANS machine, the so-called SESANS technique $[19,20]$. In this technique, the neutron wave splits not longitudinally but transversely with respect to the beam direction by a distance $\delta_{N S E}$ at the sample position. The measured quantity in this case

$$
\left\langle\sigma_{x}\right\rangle=\int d q S(q) \cos \left(q \delta_{N S E}\right) \sim I\left(\delta_{N S E}\right),
$$

where $S(q)$ is the scattering function and $I\left(\delta_{N S E}\right)=\left\langle\rho\left(\vec{r}_{i}\right) \rho\left(\vec{r}_{i}\right.\right.$ $\left.\left.+\delta_{N S E} \vec{i}_{x}\right)\right\rangle$ is the projection of the density-density correlation function on one of the axes perpendicular to the incident beam.

When NRSE with $\rho=1$ is used to realize SESANS, the measured quantity becomes a function of both space and time:

$$
\left\langle\sigma_{x}\right\rangle=\int d q S(q, \omega) \cos \left(q \delta_{N S E}\right) \cos \left(\omega \tau_{N S E}\right) \sim I\left(\delta_{N S E}, \tau_{N S E}\right),
$$

where $I\left(\delta_{N S E}, \tau_{N S E}\right)=\left\langle\rho\left(\vec{r}_{i}, t_{i}\right) \rho\left(\vec{r}_{i}+\delta \vec{i}_{x}, t_{i}+\tau_{N S E}\right)\right\rangle$. One of the applications of the experimental technique described in this paper may be found if one would use the phase coils and the gradient field of the NR spin flippers to change the parameters $\delta_{N S E}$ and $\tau_{N S E}$ independently, thus studying, for example, the dynamics of the system on the characteristic distance $\delta_{N S E}$.

Application of the four-wave SE to SESANS technique leads to the combination of the four neutron waves after their interaction with the sample. In this case, the polarization of the beam in a direction perpendicular to the quantization axis may be given as

$$
\begin{aligned}
\left\langle\sigma_{x}\right\rangle & =\int d \omega \sum_{n} S(q, \omega) \cos \left(q \delta_{N S E}^{n}\right) \cos \left(\omega \tau_{N S E}^{n}\right) \\
& \sim \sum I\left(\delta_{N S E}^{n}, \tau_{N S E}^{n}\right),
\end{aligned}
$$

where $\delta_{N S E}^{n}$ and $\tau_{N S E}^{n}$ are the transverse distance and the time delay, respectively, between different split neutron waves at the sample position ( $\mathrm{DC}^{u p}, \mathrm{DC}^{\text {down }}, \mathrm{RF}^{u p}$, and $\mathrm{RF}^{\text {down}}$ ). Such a composite correlation function may be useful in the study of periodical structures near a phase transition. In such an experiment the distance or delay-time between neutron waves may be adjusted to the periodicity of the structure under study. This may enhance a desirable contribution to the scattering and suppress an unwanted one. Further details of the applications for four-wave NRSE are currently under study. 


\section{CONCLUDING REMARKS}

(1) In this paper we demonstrated the possibility to realize spin echo of four different neutron waves or wave packets, which are produced by the neutron-resonance spin flipper in a typical neutron-resonance spin-echo setup. In order to focus all waves or wave packets in a single echo, one needs to install at least two "independent" compensation coils. The independence of the coils implies that they affect rf and dc spin-echo patterns differently. When the coils are located one between two neutron resonance spin flippers of the first SE arm and another between two SE arms of the setup, then the condition of the independence is fulfilled. The magnetic fields composing NRSF with adiabatic passage can be used for an additional adjustment.

(2) At the moment the four-wave neutron-resonance spin echo is realized on the existing setup using a standard scheme: two NRSF with $\rho=1 / 2$ is one SE arm and other two NRSF with $\rho=1 / 2$ is the second echoing SE arm. As a consequence, the amplitude of the SE pattern is $1 / 3$ of the initial polarization. Another scheme may be proposed, where the amplitude of the four-wave SE pattern is as high as the initial polarization. In this scheme, the first SE arm is built of three NRSF at equal distance from each other with spin-flip probability $\rho_{1}=1 / 2, \rho_{2}=1$, and $\rho=1 / 2$. This allows one to split a neutron into four waves and then focus them partially. The second SE arm consists of two NRSF with $\rho_{4}=1$ and $\rho_{5}=1$ in addition to the first three units. Such scheme, or its analog, should be realized to improve the signal to noise ratio of the setup.

(3) In contrast to the standard SE technique, which probes the sample properties on a length scale $R_{1}$ (or time scale $t_{1}$ ), the four-wave spin echo described above splits a neutron into four waves in the first SE arm, thus scanning the sample simultaneously on the length scale at $R_{1}, R_{2}, R_{3}$ (or at the same point on the time scale at $\left.t_{1}, t_{2}, t_{3}\right)$. When all four neutron waves are combined in the single spin echo, one can measure a composite correlation function. The first candidate for such a study is the hard sphere system. It was recently shown that the pair-correlation function in these systems is well visualized and can be directly measured by spin-echo SANS [21]. Application of the four-wave NRSE instead of ordinary SE for SANS is, therefore, very much desired in order to measure directly this composite correlation function.

\section{ACKNOWLEDGMENTS}

This work is part of the research program of the "Stichting voor Fundamenteel Onderzoek der Materie (FOM)," which is financially supported by the "Nederlandse Organisatie voor Wetenschappelijk Onderzoek (NWO)." The work was partly supported by INTAS foundation (Grant No. INTAS-03-51-6426). One of the authors (S.V.G.) thanks (Project No. SS-1671.2003.2) the Russian State Program "Neutron Research of the Condensed State."
[1] R. Golub and R. Gähler, Phys. Lett. A 123, 43 (1987).

[2] R. Gähler and R. Golub, J. Phys. (Paris) 49, 1195 (1988).

[3] R. Golub, R. Gähler, and T. Keller, Am. J. Phys. 62, 779 (1994).

[4] F. Mezei, Z. Phys. 225, 146 (1972).

[5] S. V. Grigoriev, W. H. Kraan, F. M. Mulder, and M. Th. Rekveldt, Phys. Rev. A 62, 063601 (2000).

[6] F. M. Mulder, S. V. Grigoriev, W. H. Kraan, and M. Th. Rekveldt, Europhys. Lett. 51, 13 (2000).

[7] R. Gähler, R. Golub, H. Habicht, T. Keller, and J. Felber, Physica B 229, 1 (1996).

[8] R. Gähler, J. Felber, F. Mezei, and R. Golub, Phys. Rev. A 58, 280 (1998).

[9] G. Badurek, H. Rauch, and J. Summhammer, Physica B \& C 151, 82 (1988).

[10] G. Badurek, H. Rauch, and J. Summhammer, Phys. Rev. Lett. 51, 1015 (1983).

[11] H. Rauch and S. A. Werner, Neutron Interferometry (Oxford University Press, New York, 2000).

[12] S. V. Grigoriev, R. Kreuger, W. H. Kraan, F. M. Mulder, and
M. Th. Rekveldt, Phys. Rev. A 64, 013614 (2001).

[13] W. H. Kraan, S. V. Grigoriev, M. Th. Rekveldt, W. G. Bouwman, and O. Uca, Appl. Phys. A: Mater. Sci. Process. 74, 79 (2002).

[14] V. F. Ezhov, S. N. Ivanov, V. M. Lobashev, V. A. Nazarenko, G. D. Porsev, O. V. Serduk, A. P. Serebrov, and R. R. Tal'daev, Pis'ma Zh. Tekh. Fiz. 24, 39 (1976).

[15] A. N. Bazhenov, V. M. Lobashev, A. N. Pirozhkov, and V. N. Slusar, Nucl. Instrum. Methods Phys. Res. A 332, 534 (1993).

[16] S. V. Grigoriev, A. I. Okorokov, and V. V. Runov, Nucl. Instrum. Methods Phys. Res. A 384, 451 (1997).

[17] N. F. Ramsey, Phys. Rev. A 48, 80 (1993).

[18] N. F. Ramsey, Molecular Beams (Oxford University Press, Oxford, 1990).

[19] T. Keller, R. Gähler, H. Kuntze, and R. Golub, Neutron News 6, 16 (1995).

[20] M. Th. Rekveldt, Nucl. Instrum. Methods Phys. Res. B 114, 336 (1996).

[21] T. Krouglov, I. M. de Schepper, W. G. Bouwman, and M. Th. Rekveldt, J. Appl. Crystallogr. 36, 117 (2003). 